\title{
Kinetics of Rubidium-82 After Coronary Occlusion and Reperfusion \\ Assessment of Patency and Viability in Open-chested Dogs
}

Richard A. Goldstein

Division of Cardiology and Positron Diagnostic and Research Center, The University of Texas

Health Science Center at Houston, Houston, Texas 77225-0708

\begin{abstract}
Currently available noninvasive techniques are unable to rapidly assess artery patency and tissue viability during acute myocardial infarction. In prior studies, rubidium-82 (Rb-82), a shortlived positron emitter obtained from a generator, was validated as an indicator of flow with a model that included the rate constants for transfer into and out of the cell. Accordingly, in the current study, 20 open-chested dogs with experimental infarction were studied serially at base line, after coronary occlusion, and at reperfusion. Time-activity curves acquired with beta probes on the epicardial surface were used to measure flow and net transfer of rubidium. Flow decreased to $0.41 \pm 0.08$ $\mathrm{ml} / \mathrm{min}$ per gram during occlusion and increased to $2.73 \pm 0.56$ $\mathrm{ml} / \mathrm{min}$ per gram in potentially viable ischemic tissue, whereas flows were $0.32 \pm 0.08$ during occlusion $(P<0.05$ vs. viable) and $1.58 \mathrm{ml} / \mathrm{min}$ per gram $(P<0.002$ vs. viable $)$ in irreversibly injured tissue.
\end{abstract}

The transfer rate constant for $\mathbf{R b}-82, \mathbf{k}_{\mathrm{T}}$, at base line was $+1.22 \pm 0.60 \times 10^{-3} \mathrm{~s}^{-1}$ and did not change significantly during occlusion in viable vs. nonviable samples $(+1.41 \pm 1.27$ vs. $+0.93 \pm 1.51 \times 10^{-3} \mathrm{~s}^{-1}$, respectively), except that 4 out of 11 nonviable tissue samples had negative $k_{T} s$. At reperfusion, viable myocardial samples were all positive $(+1.26 \pm 1.58$ $\left.\times 10^{-3} \mathrm{~s}^{-1}\right)$, whereas all irreversibly injured tissues had a negative $k_{T}$, indicating leakage of tracer $\left(-1.50 \pm 1.10 \times 10^{-3}\right.$ $\left.\mathrm{s}^{-1}, P<0.001\right)$. This study suggests that $\mathrm{Rb}-82$ time-activity curves can be useful to determine patency of an infarct related artery and potential viability after reperfusion during myocardial infarction.

\section{Introduction}

Several radiotracers have been used to estimate infarct size qualitatively using single photon emitting isotopes such as thallium-201 and technetium-99m-pyrophosphate (1-5). However, the physical properties of these tracers preclude precise quantification and their long half-lives limit them to a single study in an ischemic process that is undergoing continual evolution. Positron emitting tracers obviate the physical limitations of single photon emitters since quantification can be achieved using coincidence detection methods with positron

This paper was presented in part at the 1984 American College of Cardiology Scientific Session, Dallas, Texas, March 26, 1984.

Address reprint requests to Dr. Goldstein.

Received for publication 17 May 1984 and in revised form 30 October 1984.

J. Clin. Invest.

(c) The American Society for Clinical Investigation, Inc.

0021-9738/85/04/1131/07 \$1.00

Volume 75, April 1985, 1131-1137 emission tomographic reconstruction (6). The major limitation of positron studies has been the availability of tracers, since most applications require an on-site cyclotron.

Rubidium-82 (Rb-82) ${ }^{1}$ is a positron emitting isotope that is distributed in tissue in a manner similar to potassium and thallium (7-9). It has a half-life of $75 \mathrm{~s}$ and can be obtained from a commercially produced desk-top strontium-82 $\left(t_{1 / 2}\right.$ $=25 \mathrm{~d})$ generator system (10). In a recent series of studies from our group, we validated a two-compartment model for the determination of first pass extraction fraction using $\mathrm{Rb}-82$ and beta probes positioned on the epicardial surface $(11,12)$. Epicardial flow was measured accurately over a range of up to eight times normal flow and under a variety of conditions designed to alter extraction fraction.

The hypotheses of the current study are that patency of a coronary artery can be noninvasively determined based on flow measured by $\mathrm{Rb}-82$, and that irreversible injury affects myocardial cellular membrane function, which can be noninvasively detected using time-activity curves from $R b-82$.

\section{Methods}

Animal preparation. 20 fasted mongrel dogs were anesthetized with sodium pentobarbital $(30 \mathrm{mg} / \mathrm{kg})$, intubated, and ventilated with room air by a volume respirator (Harvard Apparatus Co., Inc., S. Natick, MA). Arterial pressure was monitored through a transfemoral aortic catheter and lead II of an electrocardiogram was monitored using standard subcutaneous leads. A left thoracotomy was performed in the fifth intercostal space and the heart was suspended in a pericardial cradle. The left anterior descending coronary artery (LAD) was isolated just distal to the first diagonal branch, where a snare occluder was placed. A heparinized catheter was then inserted into the aorta via the right carotid artery and connected to a withdrawal pump (Harvard Apparatus Co., Inc.).

Positron radioactivity was measured using a pair of collimated beta radiation detectors (1-cm diam) positioned on the left ventricular epicardial surface between the LAD and second diagonal branches (11, 12). One probe was faced with $1 \mathrm{~mm}$ of lead to allow background gamma radiation correction. The epicardial position of the unshielded probe was marked with methylene blue at the beginning of the experiment. The detected positron counts were processed by a pulseheight analyzer (EG \& G Ortec, Oak Ridge, TN) connected on-line to a Vax 11-780 computer that generated time-activity curves corrected for physical decay of Rb-82.

Experimental protocol. $2-3 \mathrm{mCi}$ of $\mathrm{Rb}-82$ in $10 \mathrm{ml}$ of normal saline were eluted from a Sr- $82 \rightarrow \mathrm{Rb}-82$ generator and injected as a bolus into a femoral venous catheter immediately followed by a 10 $\mathrm{cm}^{3}$ normal saline flush. An arterial sample was withdrawn at a

1. Abbreviations used in this paper: $\mathrm{k}_{1}$, rate constant into the cellular space; $k_{2}$, rate constant out of the cellular space; $k_{T}$, rate constant for net transfer of rubidium-82; LAD, left anterior descending coronary artery; NAD, nicotinamide adenine dinucleotide; $\mathrm{Rb}-82$, rubidium-82; TTC, triphenyl tetrazolium chloride; TTC-, TTC negative; TTC + , TTC positive. 
constant rate by a withdrawal pump (Harvard) to serve as an arterial reference for flow and uptake measurements.

After base-line recordings of rubidium time-activity curves, coronary occlusion was produced for one $(n=8)$, three $(n=4)$, or six $(n=8)$ followed by reperfusion after one of these three periods of occlusion by release of the snare occluder. Rubidium time-activity curves were obtained at base line, immediately after reperfusion, and $30 \mathrm{~min}$ after reperfusion in all animals.

Animals were then killed with an overdose of barbiturates and their hearts were excised. The patency of the LAD was confirmed by visual inspection and the heart was then sectioned into $1-\mathrm{cm}$ axial slices. These samples were incubated with triphenyl tetrazolium chloride (TTC) to determine histochemically whether the tissue under the unshielded beta probe had been reversibly (deep red staining) or irreversibly injured (pale staining) as previously described (13-15). Since the range of the positron particles recorded by the beta probe is only 3-4 mm, only the $4 \mathrm{~mm}$ of myocardium under the unshielded probe was used for comparison with $\mathrm{Rb}-82$ measurements (16). Areas were reported as TTC negative (TTC-) if there was evidence of irreversible damage within this sample.

Analysis of rubidium time-activity curves. Beta probes have a high detection efficiency for positron particle radiation and a low efficiency for gamma photon radiation (11). To obtain positron radioactivity per se, the time-activity curve obtained with the lead-faced beta detector (gamma radiation only) was subtracted by computer from the detector without lead (positron and gamma radiation). Regional myocardial positron radioactivity was recorded at 1-s intervals for $150 \mathrm{~s}$. The same probes were then placed over a $150-\mathrm{ml}$ beaker $(5.5-\mathrm{cm}$ diam) containing the arterial reference sample for the same interval to determine the integrated delivered dose. Since the surface area of the beaker is much greater than the probe diameter, the geometry and sample volume of blood and myocardium can be considered the same.

Myocardial flow was determined using the following equation (17):

$\mathrm{F}=\mathrm{U}(\mathrm{T}) / \mathrm{E} \cdot \int_{0}^{\mathrm{T}} \mathrm{C}_{\mathrm{a}}(t) \mathrm{d} t$

where $\mathrm{F}$ is myocardial flow (milliliters per minute per gram), $\mathrm{C}_{\mathrm{a}}$ is the arterial concentration of $R b-82$ (counts per second per milliliter), $U(T)$ is the myocardial uptake of rubidium (counts per second per gram), and $E$ is the extraction fraction of tracer. Extraction fraction was measured using a previously developed two-compartment model (11, 12). Myocardial uptake reported in the results presents the positron radioactivity in the myocardium at $150 \mathrm{~s}$ divided by the delivered dose (i.e., the integrated arterial reference radioactivity). The value is equivalent to the product of flow and extraction and the units are therefore milliliters per minute per gram $\times \mathrm{E}^{-1}$.

The rate constant for net transfer of $R b-82, k_{T}$, was obtained from a best fit monoexponential least squares regression equation applied to the probe counts from 60 to $150 \mathrm{~s}$ after administration of tracer.

Microsphere determined flow. Epicardial blood flow at occlusion and reperfusion was measured simultaneously with radioactive microspheres, 15-mm diam, labeled with $\mathrm{Sr}-85$, $\mathrm{Co}-57$, or $\mathrm{Nb}-95$. Microspheres were administered over 20-30 s into the left atrium, and an arterial reference sample (the same used for rubidium measurements) was withdrawn for $150 \mathrm{~s}$ at a rate of $16 \mathrm{ml} / \mathrm{min}$ from the ascending aorta into a preweighed syringe. At the conclusion of the experiment, an epicardial biopsy in the same sample volume as the beta detector was trimmed free of fat, and weighed. Tissue samples were counted separately with a sodium iodide well counter and a 1,000-channel pulse-height analyzer. A computer program was used to calculate flow using a least-squares analysis of the spectra $(18,19)$. Control microspheres were not performed since the $\mathrm{Rb}-82$ flow measurements have been previously validated.

Statistics. The relationship between microsphere flow and rubidium flow was determined by linear regression analysis. Differences in the rubidium transfer rate constants for viable and nonviable samples were determined with a two-tailed Student's $t$ test for unpaired samples or a Wilcoxon test where appropriate. All values reported are mean \pm SD.

\section{Results}

Rubidium uptake after coronary occlusion and reperfusion. Epicardial uptake in the LAD distribution averaged $0.72 \pm 0.15$ at base line. There were no differences in control uptake between samples that were subsequently TTC positive (TTC + ) $(n=9)$ or TTC $-(n=11)$ at necropsy (Fig. 1). Uptake of rubidium decreased after coronary occlusion to $0.15 \pm 0.06$ in samples that were TTC - at necropsy compared with $0.36 \pm 0.19$ in TTC + samples $(P<0.01)$. Reperfusion resulted in increased uptake in both groups but was less marked in TTC- samples $(0.52 \pm 0.16$ vs. $0.85 \pm 0.34, P<0.01)$. Whereas the difference between occlusion and reperfusion rubidium uptake for each sample was significant, uptake alone did not provide a clear discriminator of patency between the occlusion TTC + group and the reperfused TTC- group.

Rubidium flow after coronary occlusion and reperfusion (Fig. 2). Epicardial flow by Rb-82 was $2.04 \pm 0.70$ and $1.88 \pm 0.86$ $\mathrm{ml} / \mathrm{min}$ per gram at base line in samples that were TTC + and TTC - , respectively, at necropsy $(P=\mathrm{NS})$. Occlusion decreased flow to $0.41 \pm 0.08 \mathrm{ml} / \mathrm{min}$ per gram in TTC + samples and to $0.32 \pm 0.08 \mathrm{ml} / \mathrm{min}$ per gram in TTC - samples $(P<0.05$ TTC + vs. TTC,$- P<0.001$ vs. base line for both). At reperfusion, flow increased significantly in both regions but less in TTC- samples that paralleled the changes noted in uptake of rubidium. Flow increased to $2.73 \pm 0.56$ in TTC + samples but only to $1.58 \pm 0.78 \mathrm{ml} / \mathrm{min}$ per gram in TTC - samples $(P$ $<0.002$ ). Similar values were obtained by microspheres $(2.89 \pm 0.55$ and $1.70 \pm 0.78 \mathrm{ml} / \mathrm{min}$ per gram, respectively). These differences in flow are consistent with a component of the no-reflow phenomenon in irreversibly injured tissue samples (20). In all animals, flows by $\mathrm{Rb}-82$ exceeded $1.0 \mathrm{ml} / \mathrm{min}$ per gram after reperfusion, whereas flow was $\leq 0.5$ in all samples during occlusion. Thus, in contrast to changes in rubidium uptake, rubidium flow measurements provided a good indicator of reflow.

Transfer rate constant after occlusion and reperfusion. Fig. 3 illustrates a time-activity curve for $\mathrm{Rb}-82$ at control and

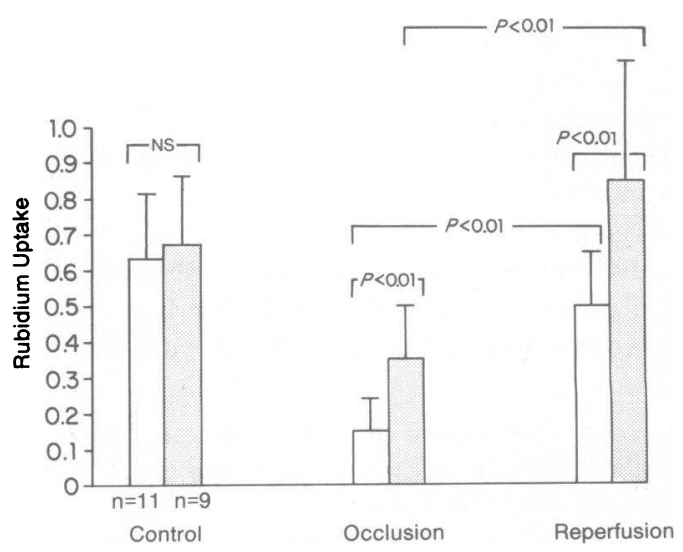

Figure 1. Epicardial uptake of Rb-82 (normalized for delivered dose) at base line and after occlusion and reperfusion for potentially viable $(\mathrm{TTC}+)$ and irreversibly injured samples (TTC-). $\square$, TTC + ; $\square$, TTC-. 


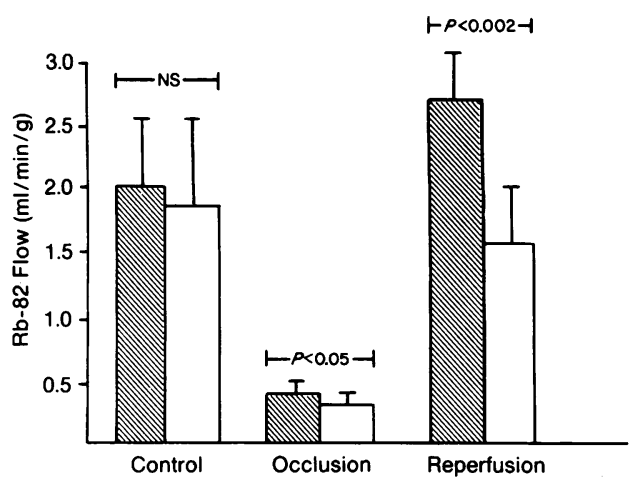

Figure 2. Epicardial flow measured by Rb-82 at base line, after occlusion, and reperfusion for potentially viable (TTC + ) and irreversibly injured samples (TTC-). $₫$, TTC+;, , TTC-

after coronary occlusion. The transfer rate constant for both examples is positive, indicating that tissue uptake of radioactivity exceeds egress. The average $\mathrm{k}_{\mathrm{T}}$ at control was $+1.22 \pm 0.60$ $\times 10^{-3} \mathrm{~s}^{-1}$ and did not significantly change during occlusion, $+1.41 \pm 1.27 \times 10^{-3} \mathrm{~s}^{-1}$ for TTC+ and $+0.93 \pm 1.51 \times 10^{-3} \mathrm{~s}^{-1}$ for TTC - tissue. 4 of the 11 TTC - tissues had negative $k_{T} s$, whereas all of the TTC + tissue had positive $\mathrm{k}_{\mathrm{T}} \mathrm{s}$. The relatively low levels of activity and therefore a low signal-to-noise ratio during occlusion in myocardium that was TTC- contributed to the large standard deviation observed in $k_{T}$ for these samples.

Fig. 4 depicts time-activity curves of $\mathrm{Rb}-82$ obtained in potentially viable (TTC + ) and irreversibly injured (TTC-) tissue following reperfusion. The TTC + tissue had a positive $k_{T}$, whereas the irreversibly injured tissue had a negative $k_{T}$. Fig. 5 presents the $\mathrm{k}_{\mathrm{T}} \mathrm{S}$ for both groups in comparison to control $\mathrm{k}_{\mathrm{T}}$. The average $\mathrm{k}_{\mathrm{T}}$ was $+1.26 \pm 1.58 \times 10^{-3} \mathrm{~s}^{-1}$ for TTC + and $-1.50 \pm 1.10 \times 10^{-3} \mathrm{~s}^{-1}$ for TTC - tissue. Furthermore, all TTC - tissue had negative $\mathrm{k}_{\mathrm{T}}$, indicating that leakage of rubidium from myocardium exceeded influx after the first pass of tracer.

Relation of rubidium and microsphere determined flow. The relation of epicardial flow during occlusion and reperfusion determined by $\mathrm{Rb}-82$ time-activity curves and microspheres is shown in Fig. 6. Although the correlation coefficient for this relation is high, the slope at 0.80 indicates that rubidium flow underestimates flow measured by microspheres. This result is not unexpected considering the model used in determining flow. The assumption made in the previous study is that during the first pass of tracer through the tissue, there is very little egress of tracer $(11,12)$. However, the data presented above demonstrate that irreversible damage produces leakage of tracer out of cells. This error is magnified by the value recorded for uptake at $150 \mathrm{~s}$ after tracer administration since the negative $\mathrm{k}_{\mathrm{T}}$ lowers residue detector counts, and thus, the flow determined by $\mathrm{Rb}-82$. If the irreversible tissue values are eliminated from this relation, the $r$ value improves to 0.95 and the slope to 0.88 .

\section{Discussion}

Most detection systems used for clinical perfusion imaging acquire counts with a poor temporal resolution such that
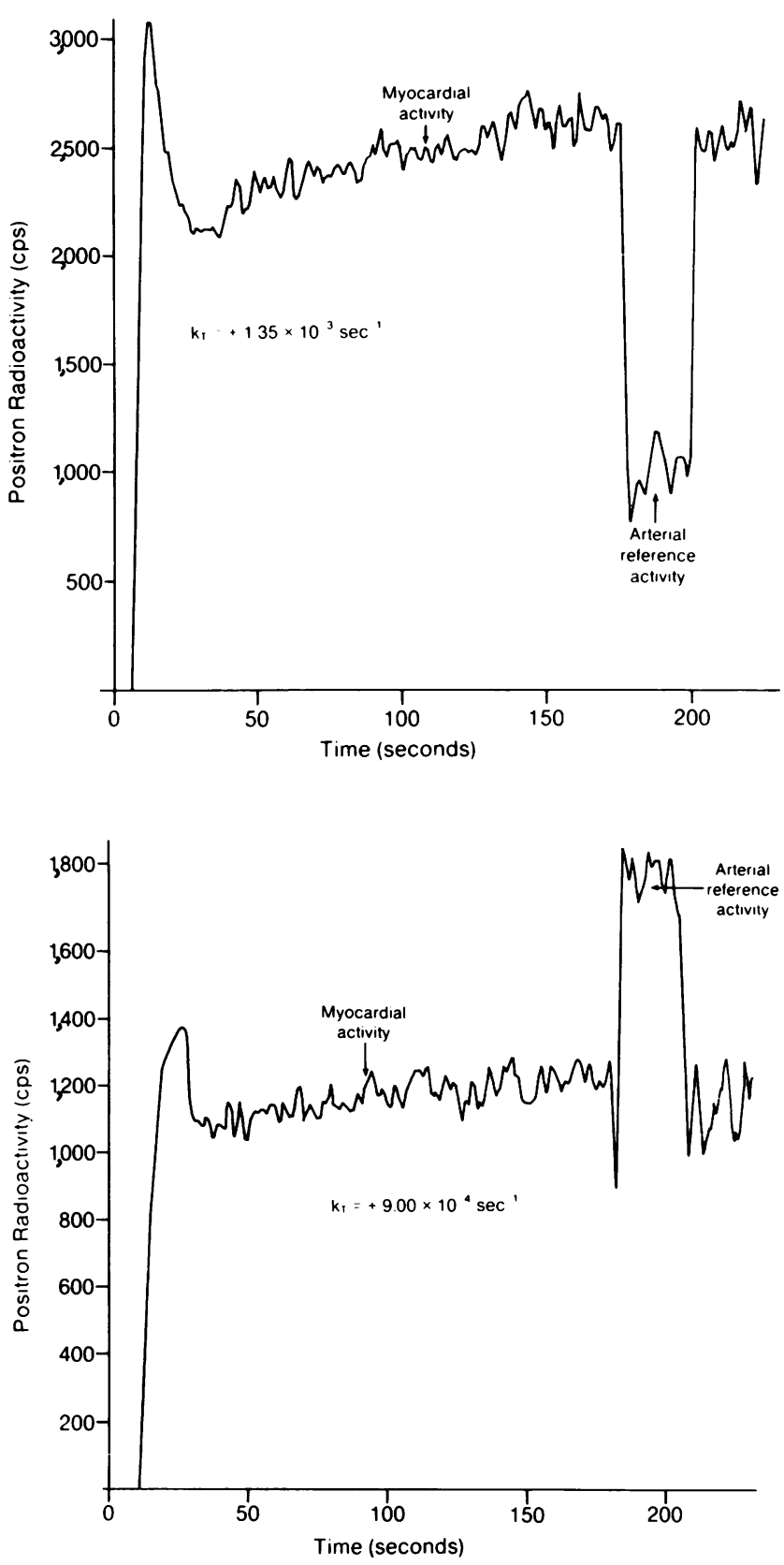

Figure 3. Epicardial time-activity curves of $\mathrm{Rb}-82$ in a control $(A)$ and after coronary occlusion $(B)$.

several minutes are required for each data point (21). The resultant count density obtained is the net of several processes including tracer delivery, extraction, and efflux, which cannot be separated into its components by standard instrumentation. Although the approach of using regional count density as an estimate of perfusion in coronary disease has been clinically employed with thallium scintigraphy, its use during acute infarction is limited since it cannot be used to differentiate reversibly from irreversibly injured myocardial regions unless redistribution images are obtained several hours later, by which time the infarct may have evolved (22). Additional problems with thallium-201 are the long half-life $\left(T_{1 / 2}=73 \mathrm{~h}\right)$ and limited spatial resolution because of variable attenuation, scatter, and random counts (21). 

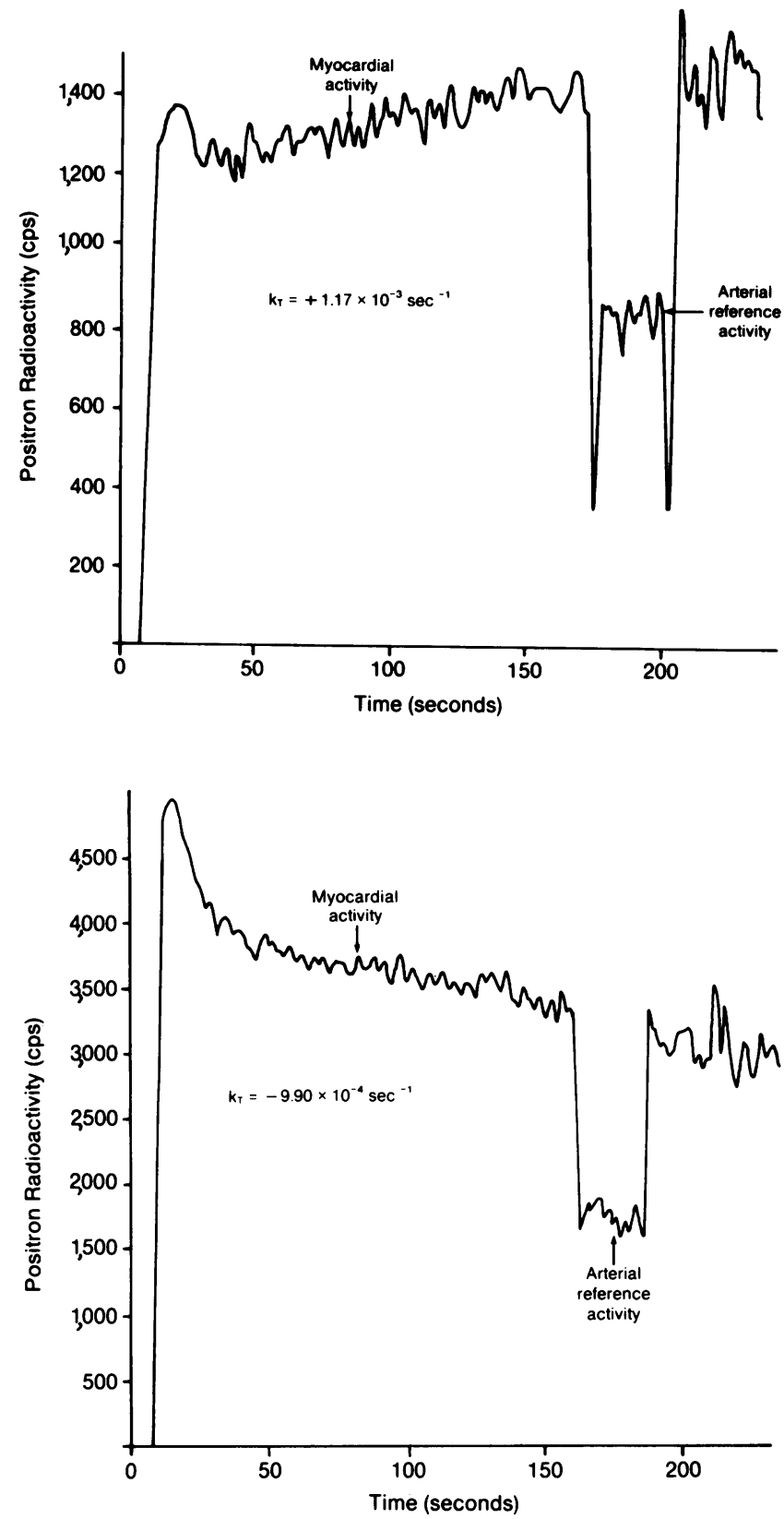

Figure 4. Epicardial time-activity curves of $\mathrm{Rb}-82$ after reperfusion in viable $(A)$ and irreversibly injured samples $(B)$.

Newer positron imaging systems have improved the temporal resolution by incorporating the time-of-flight of the photon pair, which improves the statistical accuracy of acquired counts $(23,24)$. Positron tomography has superior spatial resolution because of coincidence detection of the two photons emitted during positron decay, which limits random counts and allows quantification. In this study, the positron decay was detected, not annihilation photons. This approach allows frequent sampling of counts and is a more convenient experimental model for analysis of tracer kinetics than can be achieved with a positron camera since it avoids partial volume errors or significant motion artifacts.

Flow measurements with $R b-82$. Several potassium analogues such as thallium, rubidium, and ammonium have been used to assess regional myocardial perfusion (11, 12, 25-28). Uptake of these tracers is linearly related to flow up to 1.52.0 times resting flow, but the relationship plateaus at high flows such that uptake underestimates high flow $(12,24,29)$.

An earlier study from our group employed a two-compartment model for determination of extraction of Rb-82 during first pass of the tracer $(11,12)$. This model includes two assumptions pertinent to this paper. First, the venous egress at the time of peak counts is assumed to be minimal. For this assumption to be valid, the duration of the bolus must be shorter than the capillary transit time. A computer simulation of detected arterial and venous activity was conducted under an assumption that the venous output function was identical to the arterial input function (30). This approach resulted in a $23 \%$ underestimation of true flow. To completely validate the model experimentally, additional studies are required to determine its limitations and potential applicability.

Previous experiments have been performed to evaluate this approach as an estimate of flow including studies during acidosis and alkalosis, inhibition of the Na-K ATPase pump, $\beta$-blockade, and administration of glucose and insulin. Despite these metabolic alterations that affected membrane extraction, the model for flow was accurate within $10 \%$ of true flow since changes in extraction were determined with the model and were accompanied by similar changes in uptake. Flow by the model with $\mathrm{Rb}-82$ as the tracer was linearly related to microsphere-determined flow in the same sample volume up to eight times base line, with relatively little scatter of data.

The second assumption of the Rb- 82 flow model is that $\mathrm{Rb}-82$ leakage is negligible for the observation period of the time-activity curve (150 s). In this study, flow measurements with $\mathrm{Rb}-82$ were linearly related to microsphere flows but with considerably more scatter and more underestimation of flow than in studies in which tracer doesn't leak from cells. These results occurred for the following reasons. The assumption that there is minimal egress of Rb-82 did not hold for TTCregions during occlusion or reperfusion. It is not surprising that membrane function and thus the rate constant for egress of tracer would be significantly affected by prolonged occlusion. Reperfusion might be expected to accelerate this process because reactive hyperemia immediately after release of the occlusion may produce capillary membrane damage $(20,31)$. Thus, the measurement of myocardial uptake of Rb-82 at 150 $s$ after bolus injection would be expected to yield artifactually low measurements of flow using Rb-82 due to leakage of tracer from the field of interest. The lower net initial uptake is probably less critical since the algorithm used for extraction estimation only includes the first $30 \mathrm{~s}$ of data. Nevertheless, this change in membrane permeability would be expected to affect estimated extraction. Measuring the flow earlier, i.e., within $30 \mathrm{~s}$ of tracer injection, would still be reasonably accurate because of less leakage of rubidium from the myocardium.

Despite these limitations, the $\mathrm{Rb}-82$ flow measurements allowed distinct separation of the status of the infarct related artery with regard to reflow. The preparation used in this study did not have a stenosis and thus its relevance to clinical reperfusion remains to be tested.

Assessment of viability. There are no noninvasive methods available that allow rapid distinction between reversibly and 


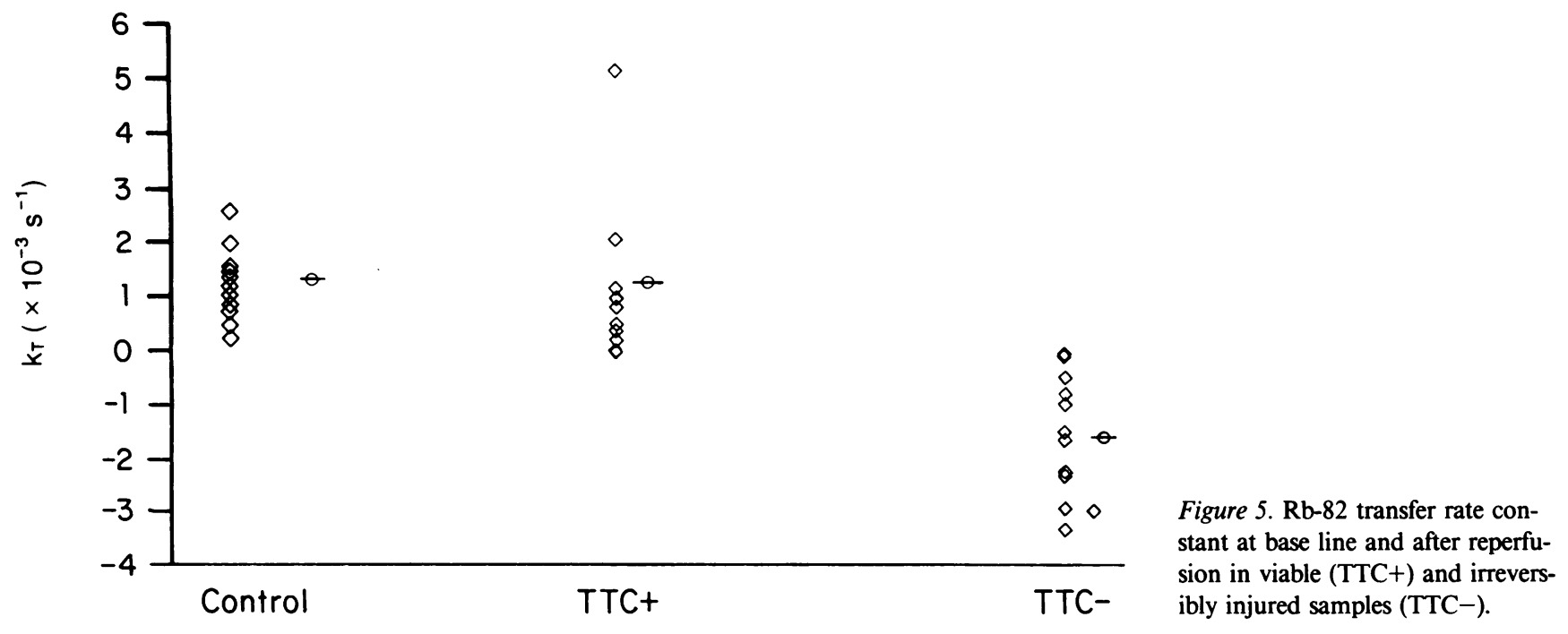

irreversibly injured ischemic myocardium. Several studies have implied that positron imaging can provide this information based on labeled carbohydrate or fatty acid metabolism, but these techniques require proximity to a cyclotron and subsequent chemical synthesis of the tracer (32-35).

In the present study, $\mathrm{Rb}-82$ uptake and flow measurements were significantly lower in TTC- regions than TTC+ regions during occlusion and reperfusion. A more specific finding associated with TTC- regions was a negative rubidium $k_{T}$ that was present in 4 out of $11(36 \%)$ during occlusion and all 11 after reperfusion. None of the TTC+ samples had a negative $\mathrm{k}_{\mathrm{T}}$ (specificity $=100 \%$ ). It seems that the mechanism responsible for a negative $k_{T}$ is the presence of membrane leakage. A negative $k_{T}$ has not been observed after any of the interventions performed in the earlier study from our group (12). Note that reversibly injured tissue was not significantly different from base-line values of $\mathbf{k}_{\mathrm{T}}$, suggesting that cellular membrane function was intact until irreversible injury occurred.

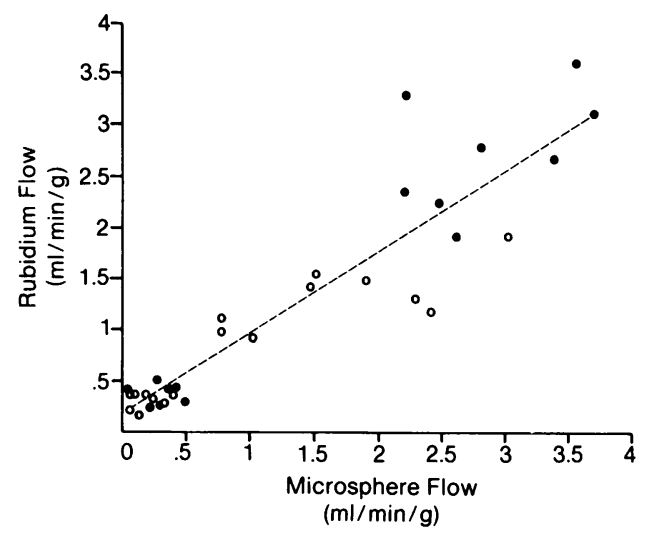

Figure 6. The relation of flow by rubidium and microsphere determined flow during ischemia and reperfusion for potentially viable (TTC + ) and irreversibly injured samples (TTC-). Rubidium flows $\geq$ $1.0 \mathrm{ml} / \mathrm{min}$ per gram were obtained after reperfusion and those $\leq 0.5$ $\mathrm{ml} / \mathrm{min}$ per gram were obtained during occlusion. (---) From equation, $\mathrm{F}_{\mathrm{Rb}}=0.19 \pm 0.10+0.80 \pm 0.06 \mathrm{~F} \mu ; \mathrm{F}_{\mathrm{Rb}}$, flow by rubidium; $\mathrm{F} \mu$, microsphere determined flow. $r=0.92 ; n=34 ; P<0.001$. $\bullet$, TTC+; o, TTC-
The observed changes in $\mathrm{k}_{\mathrm{T}}$ can be derived from the simple model shown in Fig. 7. The model consists of two compartments: an extracellular space, and cellular space along with rate constants into $\left(k_{1}\right)$ and out of $\left(k_{2}\right)$ the cellular space. The changes in counts seen by a beta probe, $\mathrm{P}$, at time $t$ can be described by the following equation:

$\frac{\mathrm{dP}(t)}{\mathrm{d} t}=\mathrm{V}_{\mathrm{E}} \mathrm{C}_{\mathrm{E}}(t) \mathrm{k}_{1}+\mathrm{V}_{\mathrm{M}} \mathrm{C}_{\mathrm{M}}(t) \mathrm{k}_{2}$,

where $\mathrm{V}$ and $\mathrm{C}$ represent the volume and concentration of radioactivity in the extracellular (E) and myocardial cellular (M) spaces, respectively, at time, $t$.

In the method used to measure flow, $\mathrm{k}_{2}$ is assumed to be negligible under control conditions. If this assumption is valid, equation (2) can be written as follows:

$\frac{\mathrm{dP}(t)}{\mathrm{d} t}=\mathrm{V}_{\mathrm{E}} \mathrm{C}_{\mathrm{E}}(t) \mathrm{k}_{1}$

Changes in probe count activity after first pass (60-150 s) would be monoexponential and thus $\mathrm{k}_{\mathrm{T}}$ would approximate $k_{1}$. This approach also seems tenable for potentially viable, ischemic, or reperfused myocardium. However, irreversible injury after reperfusion displayed a net loss of activity after first pass of the tracer indicating leakage and therefore a significant increase in $k_{2}$. If $k_{2}$ is much greater than $k_{1}$, equation (2) can be approximated as follows after first pass delivery of tracer:

$\frac{\mathrm{dP}(t)}{\mathrm{d} t}=\mathrm{V}_{\mathrm{M}} \mathrm{C}_{\mathrm{M}}(t) \mathrm{k}_{2}$.

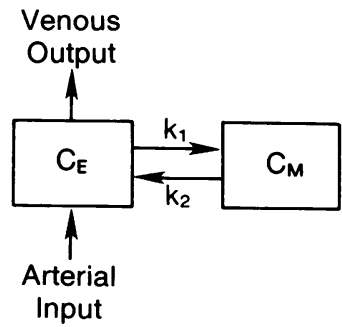

Figure 7. A two-compartment model for the concentration $(\mathrm{C})$ of $\mathrm{Rb}-82$ radioactivity into the extracellular $(\mathrm{E})$ and myocardial cellular (M) spaces including the rate constants for forward $\left(\mathrm{k}_{1}\right)$ and backwards $\left(\mathrm{k}_{2}\right)$ transfer. 
The monoexponential rate constant, $k_{\mathrm{T}}$, would thus reflect $\mathbf{k}_{\mathbf{2}}$ under these conditions. To validate this model, intracoronary injection of tracers will need to be performed to obtain a direct measurement of $\mathbf{k}_{\mathbf{2}}$.

The results must also be interpreted with regard to the reliability of TTC staining as an indicator of irreversible damage. Tetrazolium salts accept electrons in reactions catalyzed by dehydrogenases. It was originally thought that depletion of dehydrogenases was the mechanism by which failure to take up TTC indicated irreversible damage. However, recent evidence suggests that staining is dependent on nicotinamide adenine dinucleotide (NAD) since the addition of NAD or succinate restores staining in infarcted tissue (13). The mechanism by which NAD loss occurs is unclear.

TTC has been accepted as an indicator of irreversible damage by several investigators and confirmed by ultrastructural analysis (13-15). This approach may underestimate the area of necrosis if NAD has not had sufficient time to be washed out of the tissue. Reperfusion as performed in this study overcomes this potential problem (13).

The use of Rb-82 in this study offers a significant advantage over metabolic tracers because it is readily obtained from a generator and does not require additional chemical processing before administration. Background activity is insignificant after $10 \mathrm{~min}$ because of the short half-life such that serial injections could be performed to assess the effects of interventions.

Conclusion. Time-activity curves obtained using Rb-82 and beta probes can be used to determine patency of a coronary artery after experimental occlusion and reperfusion. Viability, based on the presence of TTC staining, can be determined by the $\mathbf{R b} k_{T}$, which is a measure of membrane integrity after reperfusion. Further studies with a preparation that includes a pre-existing coronary stenosis are required to test these principles before these results can be related to infarction associated with chronic stenoses in man.

\section{Acknowledgments}

The author wishes to thank Jonathan C. MacLean, who assisted with the animal preparation and data analysis, Dr. David J. Fisher for processing the microsphere data, Salma K. Marani for help with the statistical analysis, and Nizar Mullani for help with the mathematical development of the net rate constant and counting instrumentation computer services. In addition, the author appreciates the constructive comments and support of Dr. K. Lance Gould, Director of the Positron Diagnostic and Research Center (National Institutes of Health grant 2RO1 HL26862-04).

Dr. Goldstein is the recipient of a National Institutes of Health New Investigator Award (IR23HL28216-03). This work was carried out as a joint collaborative research project with the Clayton Foundation for Research, Houston, Texas and grants from the American Heart Association, National and the Texas Affiliate.

\section{References}

1. Wackers, F. J. T., E. B. Sokole, G. Samson, J. B. van der Schoot, K. I. Lie, K. L. Leim, and H. J. J. Wellens. 1976. Value and limitations of thallium-201 scintigraphy in the acute phase of myocardial infarction. N. Engl. J. Med. 295:1-5.

2. Ritchie, J. L., B. L. Zaret, H. W. Strauss, B. Pitt, D. S. Berman, H. R. Schelbert, W. L. Ashburn, H. J. Berger, and G. W. Hamilton. 1978. Myocardial imaging with thallium-201: a multicenter study in patients with angina pectoris or acute myocardial infarction. Am. J. Cardiol. 42:345-350.

3. Parkey, R. W., F. J. Bonte, S. L. Meyer, J. M. Atkins, G. L. Curry, E. M. Stokely, and J. T. Willerson. 1974. A new method for radionuclide imaging of acute myocardial infarction in humans. Circulation. 50:540-546.

4. Willerson, J. T., R. W. Parkey, J. F. Bonte, S. L. Meyer, and E. M. Stokely. 1975. Acute subendocardial infarction in patients: its detection by technetium- $99 \mathrm{~m}$ stannous pyrophosphate myocardial scintigrams. Circulation. 51:436-441.

5. Willerson, J. T., R. W. Parkey, L. M. Buja, and F. J. Bonte. 1977. Are ${ }^{99 \mathrm{~m}}$ Tc-stannous pyrophosphate myocardial scintigrams clinically useful? Clin. Nucl. Med. 2:137-145.

6. Goldstein, R. A., N. A. Mullani, and K. L. Gould. 1983. Quantitative myocardial imaging with positron emitters. In Progress in Cardiology, Vol. 12. P. N. Yu and J. R. Goodwin, editors. Lea \& Febiger, Philadelphia, PA. 147-191.

7. Donato, L., G. Bartolomei, and R. Giordani. 1964. Evaluation of myocardial blood perfusion in man with radioactive potassium or rubidium and precordial counting. Circulation. 29:195-203.

8. Ziegler, W. H., and C. A. Goresky. 1971. Kinetics of rubidium uptake in the working dog heart. Circ. Res. 29:208-220.

9. Sheehan, R. M., and E. M. Renkin. 1972. Capillary, interstitial, and cell membrane barriers to blood-tissue transport of potassium and rubidium in mammalian skeletal muscle. Circ. Res. 30:588-605.

10. Neirinckx, R. D., J. F. Kronauge, G. P. Gennaro, and M. D. Loberg. 1982. Evaluation of inorganic absorbents for the rubidium-82 generator: I. Hydrous $\mathrm{SnO}_{2}$. J. Nucl. Med. 23:245-249.

11. Mullani, N. A., R. A. Goldstein, K. L. Gould, D. J. Fisher, S. K. Marani, H. A. O'Brien, and M. D. Loberg. 1983. Myocardial perfusion with rubidium-82. I. Measurement of extraction fraction and flow with external detectors. J. Nucl. Med. 24:898-906.

12. Goldstein, R. A., N. A. Mullani, D. J. Fisher, S. K. Marani, K. L. Gould, and H. A. O'Brien. 1983. Myocardial perfusion with rubidium-82. II. The effects of metabolic and pharmacologic interventions. J. Nucl. Med. 24:907-915.

13. Schaper, J., and W. Schaper. 1983. Reperfusion of ischemic myocardium: ultrastructural and histochemical aspects. J. Am. Coll. Cardiol. 1(4):1037-1046.

14. Ellis, S. G., C. I. Henschke, T. Sandor, J. Wynne, E. Braunwald, and R. A. Kloner. 1983. Time course of functional and biochemical recovery of myocardium salvaged by reperfusion. J. Am. Coll. Cardiol. 1(4):1047-1055.

15. Fishbein, M. C., S. Meerbaum, J. Rit, U. Lando, K. Kanmatsuse, J. C. Mercier, E. Corday, and W. Ganz. 1981. Early phase acute myocardial infarct size quantification: validation of the triphenyl tetrazolium chloride tissue enzyme staining technique. Am. Heart J. 101:593-600.

16. Lerch, R. A., H. D. Ambos, S. R. Bergmann, B. E. Sobel, and M. M. Ter-Pogossian. 1982. Kinetics of positron emitters in vivo characterized with a beta probe. Am. J. Physiol. 242:H62-H67.

17. Ishii, Y., W. J. MacIntyre, W. H. Pritchard, and R. W. Eckstein. 1973. Measurement at total myocardial blood flow in dogs with ${ }^{43} \mathrm{~K}$ and the scintillation camera. Circ. Res. 33:113-122.

18. Heymann, M. A., B. D. Payne, J. I. E. Hoffman, and A. M. Rudolph. 1977. Blood flow measurements with radionuclide-labeled particles. Prog. Cardiovasc. Dis. 20:55-79.

19. Baer, R. W., E. D. Verrier, G. J. Vlahakes, B. D. Payne, and J. E. Hoffman. 1980. Validation of eight sequential myocardial blood flow determinations with radioactive microspheres using least-squares analysis. Circulation. 62(Suppl. III):III-65.

20. Kloner, R. A., C. E. Ganote, and R. B. Jennings. 1974. The "no-reflow" phenomenon after temporary coronary occlusion in the dog. J. Clin. Invest. 54:1496-1508.

21. Budinger, T. F. 1979. Physiology of physics of nuclear cardiology. 
In Nuclear Cardiology. J. T. Willerson and F. A. Davis, editors. F. A. Davis Co., Philadelphia, PA. 9-78.

22. Pohost, G. M., L. M. Air, R. H. Moore, K. A. McKusick, T. E. Guiney, and G. A. Beller. 1977. Differentiation of transiently ischemic from infarcted myocardium by serial imaging after a single dose of thallium-201. Circulation. 55:294-302.

23. Mullani, N. A., W. H. Wong, R. K. Hartz, K. Yerian, E. A. Philippe, and K. L. Gould. 1982. Design of TOFPET: a high resolution time-of-flight positron camera. In Proceedings of the International Conference on Time-of-Flight Positron Tomography. IEEE Comp. Soc., St. Louis, MO. 31-36.

24. Budinger, T. J., S. E. Derenzo, R. H. Huesman, and J. L. Cahoon. 1982. Medical criteria for the design of a dynamic positron tomograph for heart studies. IEEE Trans. Nucl. Sci. N5-29:488-492.

25. Love, W. D., and G. E. Burch. 1959. Influence of the rate of coronary plasma flow on the extraction of $\mathrm{Rb}-86$ from coronary blood. Circ. Res. 7:24-30.

26. Becker, L., R. Ferreira, and M. Thomas. 1977. Comparison of ${ }^{86} \mathrm{Rb}$ and microsphere estimates of left ventricular bloodflow distribution. J. Nucl. Med. 15:969-973.

27. Goldstein, R. A., M. S. Klein, M. J. Welch, and B. E. Sobel. 1980. External assessment of myocardial metabolism with ${ }^{1} \mathrm{C}$-palmitate in vivo. J. Nucl. Med. 21:342-348.

28. Schelbert, H. R., M. E. Phelps, E. J. Hoffman, S.-C. Huan, C. E. Selin, and D. E. Kuhl. 1979. Regional myocardial perfusion assessed with $\mathrm{N}-13$ labeled ammonia and positron emission computerized axial tomography. Am. J. Card. 43:209-218.
29. Schelbert, H. R., W. L. Ashburn, D. M. Chauncey, and S. E. Halpern. 1977. Comparative myocardial uptake of intravenously administered radionuclides. J. Nucl. Med. 15:1092-1100.

30. Mullani, N. A., and K. L. Gould. 1984. Reply to Re: first-pass measurements of regional blood flow with external detectors (letter). J. Nucl. Med. 25:830-836.

31. Bresnahan, G. F., R. Roberts, W. E. Shell, J. Ross, and B. E. Sobel. 1974. Deleterious effects due to hemorrhage after myocardial reperfusion. Am. J. Cardiol. 33:82-86.

32. Ter-Pogossian, M. M., M. S. Klein, M. S. Markham, R. Roberts, and B. E. Sobel. 1980. Regional assessment of myocardial metabolic integrity in vivo by positron-emission tomography with ${ }^{11} \mathrm{C}$ labeled palmitate. Circulation. 61:242-255.

33. Lerch, R. A., H. D. Ambos, S. R. Bergmann, M. J. Welch, M. M. Ter-Pogossian, and B. E. Sobel. 1981. Localization of viable, ischemic myocardium by positron-emission tomography with ${ }^{11} \mathrm{C}$ Palmitate. Circulation. 64:689-699.

34. Lerch, R. A., S. R. Bergmann, H. D. Ambos, M. J. Welch, M. M. Ter-Pogossian, and B. E. Sobel. 1982. Effect of flow-independent reduction of metabolism on regional myocardial clearance of ${ }^{11} \mathrm{C}$ Palmitate. Circulation. 65:731-738.

35. Marshall, R. C., J. H. Tillisch, M. E. Phelps, S. Huang, R. Carson, E. Henze, and H. R. Schelbert. 1983. Identification and differentiation of resting myocardial ischemia and infarction in man with positron computed tomography, ${ }^{18} \mathrm{~F}$-labeled fluorodeoxyglucose and N-13 ammonia. Circulation. 67:766-778. 